\title{
Utilization of a zero power reactor for examination of operator influence on reactor control
}

\author{
Tomas Bily \\ Dept. of Nuclear Reactors, FNSPE \\ Czech Technical University in Prague \\ Prague, Czech Republic \\ tomas.bily@fjfi.cvut.cz
}

\author{
Alice Darbyshire \\ Nuclear Faculty \\ Defence Academy of the United \\ Kingdom \\ Gosport, United Kingdom \\ alice.m.darbyshire@gmail.com
}

\author{
Ondrej Huml \\ Dept. of Nuclear Reactors, FNSPE \\ Czech Technical University in Prague \\ Prague, Czech Republic \\ ondrej.huml@fjfi.cvut.cz
}

\begin{abstract}
The reactor operator is an inseparable part of reactor safety and his actions influence the course of the reactor transients. Understanding the operator's effect on reactor control thus contributes to the knowledge of the safe operation of nuclear reactors. Complementary to studies performed on simulators, or utilizing standard reactor or plant operational data, the dedicated experiments at research reactors can help to improve the insight into human aspects of reactor control. The potential of research reactors to study the human operator aspects of reactor control is broad; the related activities thus may also help to increase the utilization of research reactor facilities.
\end{abstract}

The paper summarizes the considerations that had to be assessed during the implementation of such experiments at the VR-1 research reactor from the viewpoint of the reactor facility. Further, it recapitulates the activities that have been performed in this field at the VR-1 reactor in the collaboration of the Czech Technical University in Prague with the Defence Academy of the United Kingdom in the previous years.

Keywords- operator effect, reactor control, reactor dynamics, zero power reactor, control engineering

\section{INTRODUCTION}

Analyses of nuclear reactor transients are usually performed solely by codes describing the time dependent behaviour of a nuclear reactor as a physical system. However, in reality the course of a reactor transient is ruled not only by reactor physics, but also by a response of a reactor operator. Understanding the influence of the reactor operator on reactor control may thus improve our knowledge of safety cases, and may serve for operators' evaluation during their training and retraining as well. The potential fields of applications thus include, among others, improvements in operator training programs, including, quantitative evaluation of an individual operator's progress, understanding the operator-reactor system dynamic response, defining the manual controllability boundaries, and the evaluation of the human-machine interface, including effects of its modifications on the system controllability [1]

Although, historically they have not been commonly used for this purpose, zero power reactors seem to be the proper tool for the related research. This paper summarizes the related considerations, tools and approaches, and the gained experience from a reactor facility perspective.

\section{VR-1 REACTOR}

The VR-1 is a zero power pool type reactor operated by the Czech Technical University in Prague. The reactor core consists of 16 to 21 fuel assemblies of IRT-4M type enriched to $19.7 \%$ of $\mathrm{U}-235$. The reactor has seven neutron absorbing rods, out of which two are used for power regulations, the remaining being the safety and shim rods. The reactor can be operated in manual or in automatic mode. In the manual mode, an operator can directly change the rod positions using dedicated buttons. In automatic mode, an operator controls the desired power and an appropriate rod movement is achieved by the control computer.

The installed human machine interface [2] enables the operator to observe the course of time dependent reactor operation parameters, such as reactor power or rod positions.

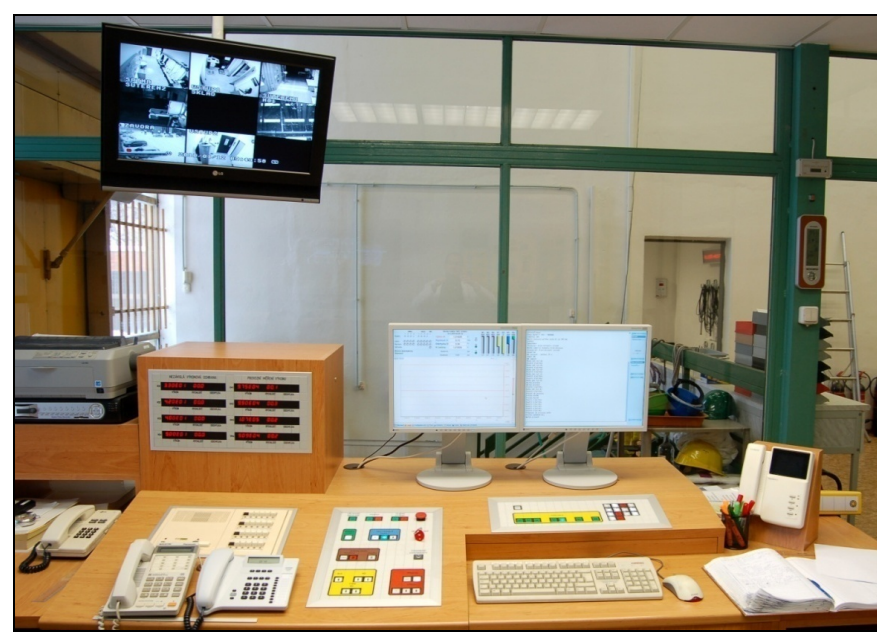

Fig. 1. Human-machine interface of the VR-1 reactor (before HMI desk upgrade in mid-2018)

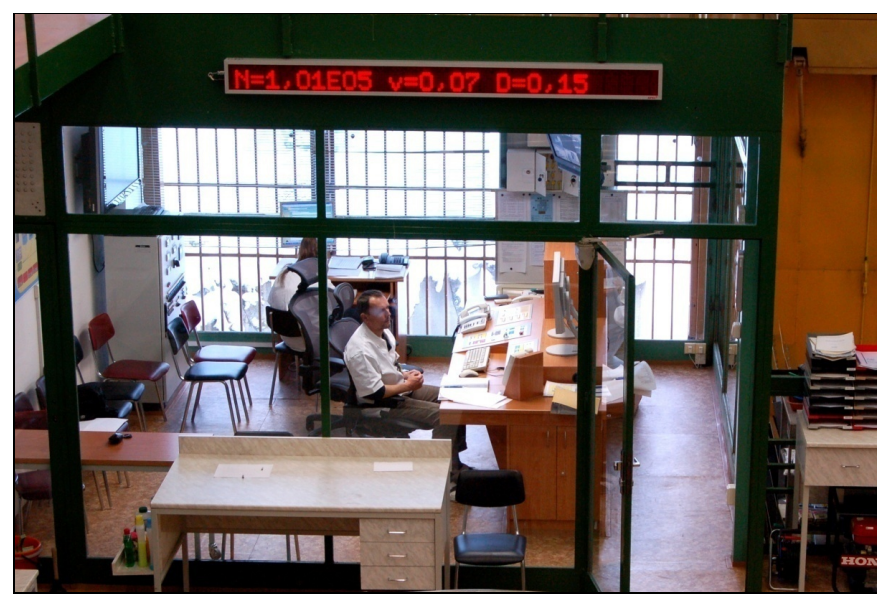

Fig. 2. Control room of the VR-1 reactor (before HMI desk upgrade in mid-2018) 


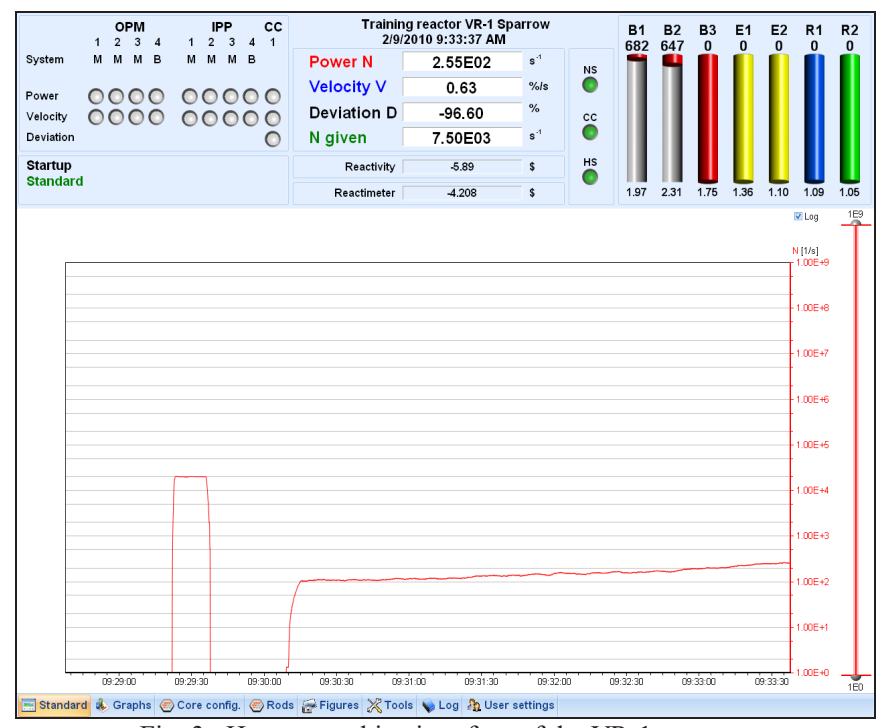

Fig. 3. Human machine interface of the VR-1 reactor

\section{EXAMINATION OF OPERATOR INFLUENCE ON REACTOR CONTROL AT THE VR-1 REACTOR}

Experiments aimed at operator aspects of reactor control have been discussed as a potential field of cooperation between the Defence Academy of the United Kingdom and the Czech Technical University in Prague since 2015, at which time the first experiments were performed. Since the beginning the experiments have been designed to utilize as much as possible of the available reactor infrastructure. The aspects which had to be considered in order to introduce the required type of operator examination included: core physics, human characteristics variations, input and output data acquisition, testing procedures, required equipment and the external conditions.

\section{A. Core physics aspects}

Studies of the operator influence on reactor control may be performed at any type of nuclear reactor. However, for initial experiments it was beneficial to run the experiments in the zero power operational region of the reactor (for the VR1 reactor this covers practically the whole power range). At zero power the core heating and core temperature changes are negligible, thus internal reactivity feedbacks are negligible as well. Hence, the core physics can be described by a simple point kinetics model relating the reactivity and the power changes; and the operator effects can be well separated from the core physics effects.

Operator performance studies utilize various reactor transients. Thus their design needs to be optimized with respect to related reactor operation limitations. In the case of the VR-1 reactor the limitations were the allowable power rate and allowable value of reactivity insertion rate. The former parameter is limited by $4 \%$ of actual power per second for warning signalization, and $6 \%$ of actual power per second for scram signalization. For the latter, it is only allowed to introduce reactivity lower than $0.1 \$$ per second. On the other hand, the studied transients have to be high enough to enable efficient data analysis, and this can be achieved by manipulation of low reactivity worth control rods. In the case of the VR-1, the control rod with a reactivity worth around $1 \$$ was used for the experiments. The rod can be moved from 0 (bottom position) to $680 \mathrm{~mm}$ with a step size of $0.25 \mathrm{~mm}$. For the tests in which external reactivity is used, the size and rate of its introduction to the core has to be assessed with respect to the above mentioned limitations.

\section{B. Methods for operator effect examination, and experimental equipment}

In principal, a zero power reactor enables two kinds of operator tests to be performed:

1) Reacting operator: the operator had to react in a prescribed way to a known, or unknown reactivity input and his response is being evaluated, or

2) Acting operator: the operator is asked to follow a prescribed input and the discrepancies between the expected and the real output are evaluated.

The first approach examines how an operator reacts to external reactivity inputs (i.e. external to those achievable by the reactor control system). To establish such kind of experiments a device that enables the production of reactivity (power) perturbations that are independent to reactor operator is needed. In the case of the VR-1 reactor experiments, the existing 'device for dynamics studies' [3] has been used. The device is based on a pneumatic system which enables fast movements of an inserted specimen (typically a piece of cadmium). The device can produce three types of movements: a) pulses, i.e. movements from the starting position to another defined position and then returning to initial position, b) steps, i.e. movements from the starting position to another defined position, and cycling, i.e. periodic movements between two defined positions, with an adjustable starting position. In all cases the speed of movement can be adjusted between $1 \mathrm{~mm} / \mathrm{s}$ up to few hundreds $\mathrm{mm} / \mathrm{s}$. For operator influence examinations the range of introducible reactivity has been optimized by the proper choice of the sample reactivity worth and the initial sample position. The optimization was aimed at producing fast and well recognizable power changes by the sample movement, which remains reasonably below the power rate warning signalization of the reactor control system. The optimal sample reactivity worth was found about $0.06 \$$. The sample speed was set to $100 \mathrm{~mm} / \mathrm{s}$. The examined operator had to react to a set of external reactivity changes produced by the device for dynamics studies using standard operational means, i.e. using control rod movement.

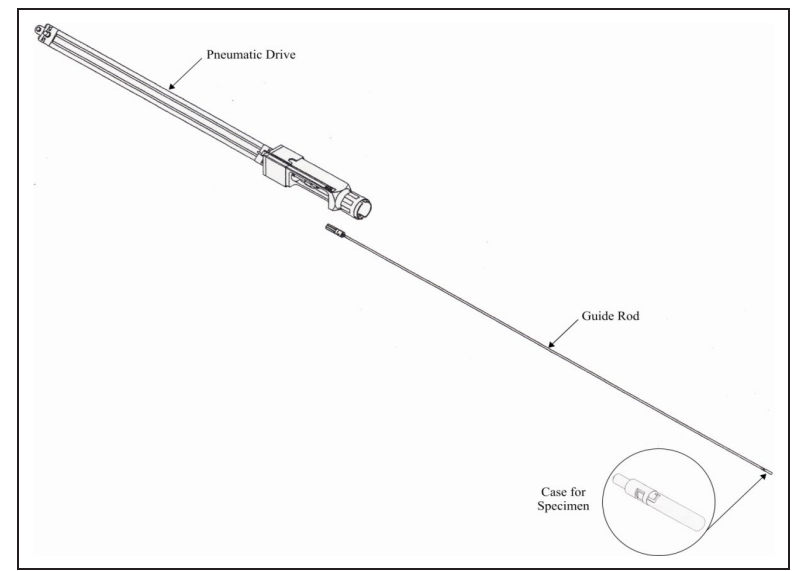

Fig. 4. Device for dynamics studies at the VR-1 reactor [4]

In the second approach, an operator has to use standard operational means, i.e. a control rod, to follow a prescribed 
input. This approach may require specific software which provides information about the desired operator actions, or the required operator actions may be given verbally, by way of an instruction or command to the operator (by a shift leader).
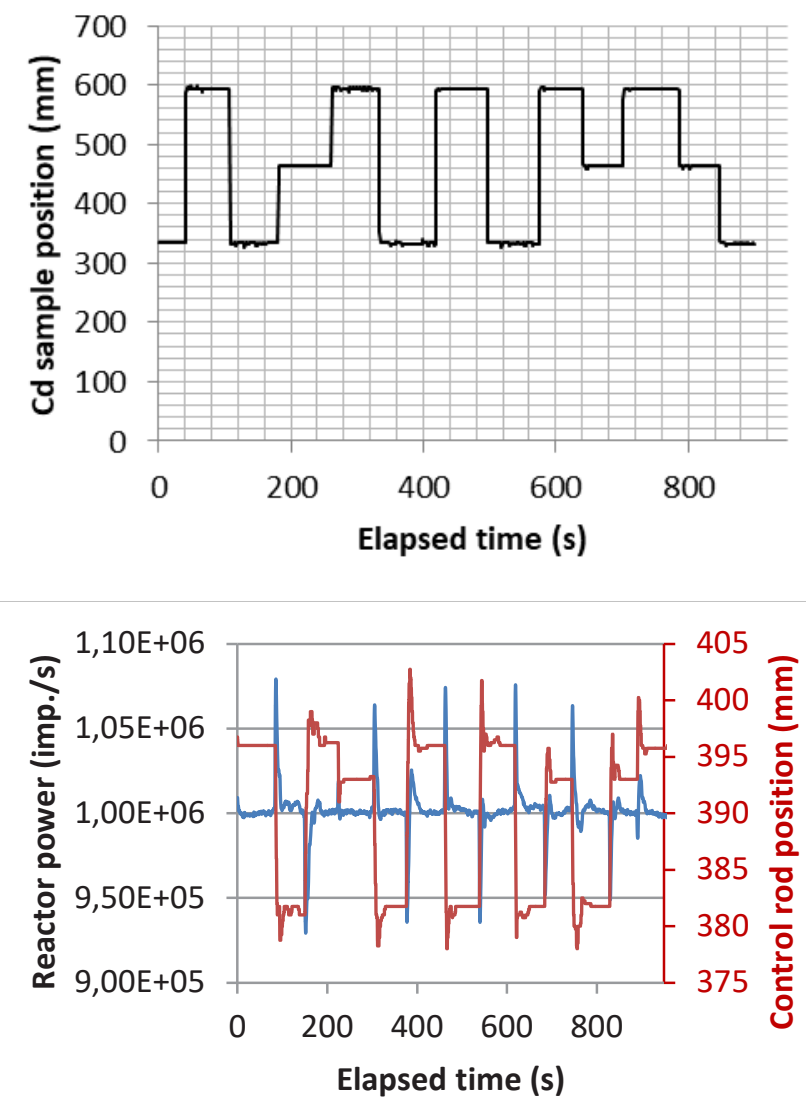

\section{- Reactor power $\quad$ Rod position}

Fig. 5. Example of a disturbance rejection test. The operator was asked to keep the power at a constant level. The device for dynamics studies provided external reactivity changes which were to be compensated by an operator using control rod movements. Up: Movement of Cadmium (Cd) sample in device for dynamics studies; Down: operator response by control rod movement and reactor power evolution. The rods can be moved from 0 to $680 \mathrm{~mm}$, and the active part of fuel assemblies is $600 \mathrm{~mm}$.

\section{Human characteristics variations}

Contrary to the core physics, the operator effects exhibit operator-to-operator variations. Thus the experiments can be aimed at either the performance evaluation of a single operator, or aimed at a set of operators, to estimate the effect of human factors dispersion on the studied operator related parameters that could influence the reactor control. Such factors may include age, experience, workload, or the professional background of the operator.

The experiments at the VR-1 reactor benefits from the fact that the facility is the university's training reactor with a large amount of licensed operators (more than 10). The operators significantly differ in their reactor control workload, professional background (either technician based or scientific based), age and experience (from a few years to more than twenty years). The facility enables students and trainees operators within their education or training sessions. Although evaluating their performance may provide valuable information for early career, low-experienced operators, they have not been tested or evaluated, thus far.

\section{Data acquisition system}

To study an operator's influence on reactor control using control engineering techniques and models one needs to have digitalized time-dependent information of the input and output parameters of operator examinations. This includes the reactor control system data, with as a minimum, the value of the reactor power and the control rod position. If external devices are used (e.g. for external reactivity changes, or for information on the desired prescribed power profile that should be followed by the operator), they need to allow for the acquisition of digital data that can be synchronised with the reactor operator data. In the case of the VR-1 reactor, the Instrumentation and Control (I\&C) system has been fully digital since the reactor was first operated in 1990. The I\&C system has since been completely upgraded in 2009 [5]. In 2011, dedicated software [6] was developed to enable the online and offline transport of selected reactor I\&C data outside the control system. Later, the system was upgraded to allow for the acquisition of data from various experimental devices, together with reactor I\&C data.

The sampling rate of the observed parameters was defined based on the needs of the data processing algorithms that were used in the $r$ control engineering models that describe the human operator behaviour. The time step of 0.1 $\mathrm{s}$ was found to be adequate for the experiments performed.

\section{E. External conditions}

The performance of the reactor operator may depend on the external conditions under which the tasks are to be performed. Thus, including variations in external conditions may help to understand the range of operator response. The external conditions should include, in particular, varying the amount of stress and the workload conditions. Obviously, studies on stress dependent operator performance have to stay within the safe practices of nuclear reactor operation. In the case of the VR-1 reactor experiments it was decided that they should correspond to situations which may typically occur during daily reactor operation. Still, the space to study stress dependent operator performance remains broad and may also include:

a) Single isolated task vs. complex task examination,

b) Response to a new, or to already known, transient,

c) Presence/absence of visual/audible information about the external reactivity change,

d) Perturbations/commands that are known in advance or are new at the time of request,

e) Full operator concentration on the task-at-hand during the examination period, or the addition of one or more distracting requirements on the operator during the examination. This may include periodic or on-demand observation and recording of selected reactor parameters.

\section{F. Other aspects}

The presence of an operator response evaluator during the experiments has been found to be important in understanding all of the behaviours and characteristics of an operator's response during individual tests, and in discovering the correlations with other factors that can influence operator response or concentration. This may 
somehow limit the capability of the facility for performing these types of tests. At the VR-1 reactor, this aspect was improved during the reactor operator desk upgrade, which was performed in mid-2018, when a videoconference system was installed in the control room to support remote education and training capabilities. The videoconference system is connected to multiple cameras inside and outside the reactor control room. Thus, video and audio signal from the reactor control room may be transmitted to remote sites, an enabling an evaluator to make on-line observations and discussions regarding the course of the operator performance experiments and, if required, a record of operation actions and experimental processes can be obtained.

\section{HISTORY OF OPERATOR INFLUENCE ON REACTOR CONTROL AT THE VR-1 REACTOR}

The development of the experiments to evaluate the operator influence on reactor control has been progressed through the close cooperation between the control engineers with their initial requests for tests and the reactor operating team who provide feedback on the feasibility of the proposed tests, and who also contribute specific knowledge of the conditions in which the operators have to work. The quality of the experimental outputs is continuously improving along with the mutual understanding of both points of view. The first discussions regarding the feasibility of performing operator experiments at the VR-1 reactor facility started in 2015. Disturbance rejection experiments, i.e. experiments in which an operator has to react to an externally induced reactivity/power change, were found to be quickly and easily implemented using the existing reactor equipment. At the end of 2015, the first experiments were performed with the aim of understanding the most important factors in the experimental design and implementation, and also to gain experience in applying control engineering methods to the real experimental data. Based on these tests, a four-stage procedure of operator tests was defined, with a gradual increase in task complexity and operator workload. Three operators of the VR-1 reactor were selected for these tests, each with a significantly different background (age, knowledge, experience, or reactor operator workload) in 2016. Duration of a single test typically varied between two and fifteen minutes. Some results have been already published in [1]. In 2017, tracking experiments i.e. experiments in which an operator is asked to follow a prescribed input were discussed and a test procedure based on a shift-leader's audible commands was developed. The procedure was tested using three reactor operators. These initial tests revealed an uncertainty in the specific timing of the audible commands, during off-line evaluation of the operators' performance. In 2018, an in-house software was developed, with the aim of decreasing this uncertainty in audible command timing. The bespoke software provides the operator with a real-time graphical display of the input signal (e.g. desired reactor power). The software will also use the reactor operation data, which is shared through the Experimental studio software [3] and it will enable a complex time-dependent desired power input to be set for the operator to follow; also it will have the capacity for on-line visualization the desired reactor power and the actual reactor power achieved by an operator.

\section{CONCLUSION}

The VR-1 reactor has been utilized for the unique examination of operator influence on reactor control since 2015. During the last few years, the capability of the facility for such studies has been proven, and most aspects of experimental setups and procedures have been understood. A significant potential to undertake related future studies has been identified.

The reported research was mainly achieved with the existing instrumentation and equipment at the facility. The identification of operator dynamic characteristics have significantly benefited from the education and training orientation of the facility. The early success of the experiments have been due to, in particular, the existing bespoke experimental instrumentation for reactor dynamics studies, the reactor operation data acquisition system, and the broad range of experienced operators at the VR-1 reactor facility.

\section{ACKNOWLEDGMENT}

The paper was supported by the project "Strengthening and development of research at Czech Technical University in Prague with the use of research infrastructure VR-1 Training Reactor for research activities" (reg. nr. CZ.02.1.010.00.016_0130001790) supported by Operational Programme Research, Development and Education cofinanced from European Structural and Investment Funds and from state budget of the Czech Republic. References

\section{REFERENCES}

[1] A.M. Darbyshire, T. Bily, J.S. Hatherall, K.D. Atkinson, "Manual control during reactivity transients at the VR-1 reactor - I. Disturbance rejection," Nuclear Engineering and Design, vol. 325, pp. 178-183, 2017, DOI:10.1016/j.nucengdes.2017.09.025.

[2] M. Kropík, J. Rataj, M. Juř́ičková "New human-machine interface at VR-1 reactor" In: 18th International Conference on Nuclear Engineering. New York: American Society of Mechanical Engineers ASME, 2010. ISBN 978-0-7918-4929-3

[3] M. Kropík, J. Rataj, "New Control and Data Acquisition of Experiments" In: Nuclear Energy for New Europe 2011. Ljubljana: Nuclear Society of Slovenia, 2011. pp. 1-12. ISBN 978-961-6207-3242011

[4] J. Rataj et al., "Reactor Physics Course at VR-1 Reactor". 2nd edition, Prague, Czech Republic, CTU in Prague, 2014.

[5] M. Kropík, M. Juříčková "Calibration of new I\&C at VR-1 training reactor, Nuclear Engineering and Design, Volume 241, Issue 4, 2011, Pages 1090-1096, ISSN 0029-5493, https://doi.org/10.1016/j.nucengdes.2010.05.049. (http://www.sciencedirect.com/science/article/pii/S002954931000375 4)

[6] J. Rataj, L. Sklenka, "New Experimental Instrumentation for Study of Reactor Dynamics at Zero Power Reactor VR-1" In: RRFM 2011 Transactions. Brusel: European Nuclear Society, 2011. pp. 177-181. ISBN 978-92-95064-11-9. 